\title{
Manganese: the Controversial Metal. At What Levels Can Deleterious Effects Occur?
}

Manganese $(\mathrm{Mn})$ is of particular importance to Canadians due to the inclusion, since 1977, of an organomanganese compound (MMT) as an antiknock agent and to enhance octane in unleaded gas in this country. In the United States, the Environmental Protection Agency (EPA) opposed approval of MMT on the grounds that critical information is lacking on exposure and dose and on low level, chronic toxicity of manganese to humans. However, this decision was brought before the courts and won by the manufacturers on grounds unrelated to the health issue. As of January, 1996, it is permitted for use in the United States. The Environmental Defense Fund, a Washington-based group representing 300,000 members, along with 36 other advocacy groups have called upon the gasoline refiners and marketers to adopt an "MMT Free" policy until more scientific information is available on the effects of this metal on human health. Other anthropogenic sources of manganese pollution in air and water include mining, industrial emissions and manganese-containing pesticides. High levels of natural manganese have been known to contaminate water supplies and food sources.

The controversy over manganese stems from the fact that we do not know at what level continuous exposure to this metal can cause adverse health effects, particularly over the long term. On the one hand, manganese is an essential element, present in metalloproteins, such as superoxide dismutase and pyruvate carboxylase and in the cytoplasmic glial enzyme, glutamine synthetase. Normally ingested through food and water, manganese is subject to delicate homeostatic control; only $1-3 \%$ of ingested $\mathrm{Mn}$ is absorbed and it is rapidly cleared from the blood by the liver and excreted in bile. Manganese deficiency in animals and humans is associated with nervous system dysfunction and convulsions; low levels of blood manganese have been reported among epileptic patients as compared to controls. ${ }^{1}$

On the other hand, high levels of manganese exposure, observed primarily among workers exposed to manganese dust, produce neurologic disorders, involving the extrapyramidal system. Manganism, described over the last 150 years among hundreds of miners and manganese alloy production workers throughout the world, bears many similarities to Parkinson's Disease; the final stages are characterized by a masked facies, resting tremor, rigidity, freezing episodes, akinesia, hypersialorrhea, but also by dystonia (particularly of smile and foot, giving way to the "cock's sign" i.e. walking on the metatarsophalangeal joint), hyperreflexia, vasomotor changes and altered mental status. Several workers, who developed manganism subsequent to the breakdown of the ventilation in a ferromanganese alloy plant in Taiwan, are currently being followed by a group of neurologists and the results of their studies and interventions are rapidly increasing our understanding of manganism, its progression and the appropriateness of therapeutic measures. ${ }^{2-4}$
At lower levels of exposure, recent epidemiological studies, have compared nervous system functioning of active workers exposed to manganese dust at levels insufficient to cause clinically apparent neurological disorders. Although there are some inter-study differences, the overall findings reveal that the exposed workers display slowing of motor functions, increased tremor, reduced response speed, and diadochokinesia, as well as impairment of certain intellectual functions and mood lability. ${ }^{5}$ These results, which are consistent with our knowledge of the action of manganese on the central nervous system, suggest that there may be a dose-related continuum from early alterations to disease. At lower doses of exposure, a form of micromanganism is observable among exposed groups; as the dose increases, clinical manifestations become evident, increasing in severity with increasing exposure. There is also concern that overexposure to manganese may accelerate neuronal attrition, thereby augmenting the risk for later life neurodegenerative disorders, particularly parkinsonism.

Manganese toxicity is thought to derive from its affinity for neuromelanin, combined with its capacity to interfere with the normal functioning of dopaminergic systems. Magnetic resonance imaging (MRI) of manganese intoxicated humans and primates have confirmed that manganese is localized preferentially in the globus pallidus, striatum and substantia nigra. Findings from studies of the pathophysiology of manganese poisoning have not always been consistent, probably due to variations in species, type of manganese administered, means and time frame of administration. The bulk of neuropathology (neuronal loss and gliosis) seems to be located at the striatum, subthalamic nucleus and pallidum, with little change at the substantia nigra, but lesions are also found throughout the cerebrum, the brain stem and the cerebellum. Most investigations note reduced concentrations of dopamine in one or more of the basal ganglia structures and a recent study of manganese intoxicated monkeys shows alterations in dopaminergic post-synaptic structures, with a reduction of $\mathrm{Dl}$ receptors in the striatum without a concomitant loss of D2 receptors. ${ }^{6}$ Different hypotheses have been put forward to explain how manganese affects dopaminergic neurotransmission, including, the formation of free radicals through glutathione reduction, decreased glutathione peroxidase activity, the auto-oxidation of dopamine via the transformation of $\mathrm{Mn2}+$ to $\mathrm{Mn3+}$, inhibition of mitochondrial respiration, abnormal hydrocarbon metabolism and excitotoxic processes through NMDA receptors.

The possible role of manganese in basal ganglia disorders secondary to liver failure has recently been brought to light by a series of very elegant studies. In this issue of the Journal, Hauser and coworkers ${ }^{7}$ (see page 95) report their findings relating blood manganese and brain MRI changes in patients with 
biopsy-proven cirrhosis. Their results, coupled to a recent publication on the subject, ${ }^{8}$ suggest that manganese homeostasis, carefully regulated by hepatic enzymes, fails in certain forms of liver dysfunction leading to high levels of blood manganese. Taken up by the basal ganglia, particularly the globus pallidus and substantia nigra, the increased levels of manganese are monitored as MRI abnormalities. Indeed, chronic hepatic encephalopathy, characterized by signs and symptoms of pyramidal and extrapyramidal dysfunction, and neuropsychiatric disorders bears many similarities to manganism.

The notion of manganese overload, raised by the Hauser and colleagues, ${ }^{7}$ is probably one of the key issues in manganese intoxication. Since more than $98 \%$ of the absorbed manganese is excreted in bile, it is possible that liver failure precludes the regular excretion, thereby increasing blood manganese levels. But is there a threshold over which the control system ceases to function or is it a gradual process with possible compensatory mechanisms? Is there a cascade of control mechanisms? Where does manganese uptake in the brain fit into this scheme? The results presented by Hauser et al. ${ }^{7}$ are similar to those reported by others ${ }^{8}$ showing very strong correlations between MRI Tl scores and blood manganese concentration. These results are not only important to the understanding of chronic hepatic encephalopathy, but may be key to comprehending what takes place with environmental exposure below levels of extreme overload.

Although blood manganese levels were significantly higher among the exposed as compared to non-exposed reference populations in reports of early nervous system dysfunction among manganese exposed workers, ${ }^{9}$ the observed levels were considerably lower than those reported by Hauser et al. ${ }^{7}$ in the cirrhotic patients (although the reference levels are in the same range). A few studies indicate that even in the presence of low level environmental or workplace manganese exposure, blood manganese levels between subjects with higher and lower exposures do not differ, although signs of mild neurological and/or neuropsychological dysfunction are present among subjects that are more highly exposed. Although in these situations, homeostatic mechanisms appear to be able to keep blood manganese within certain limits, the neurological responses may reflect a certain degree of present or past manganese overload in the brain.

One of the public health concerns about manganese related disorders is that they appear to be progressive, even when exposure has ceased. Previous case reports of progression of signs and symptoms of manganism, following cessation of exposure, have been confirmed by the studies on the Taiwanese ferro-manganese workers. ${ }^{3}$ According to the authors, ${ }^{3}$ parkinsonian symptoms showed a slow progression; review of video records confirmed worsening of parkinsonism, particularly in difficulty in turning and a highly significant increase in the mean disability scores over a four year period. A case report on the MRI of a welder with manganism, revealed that after a period of 6 months with no exposure, manganese was cleared from the basal ganglia although the neurological signs and symptoms remained. ${ }^{10}$
Hauser and colleagues ${ }^{7}$ aptly point out links between $\mathrm{Mn}$ intoxication, encephalopathy, parkinsonism and chronic liver disease. Studies of neurological manifestations associated to manganese levels in persons with hepatic disorders may be of particular importance in determining dose-effect relations, however, the role of deficiency states of calcium and magnesium, which can also be present in liver failure, must be considered.

In September, 1984, an issue of the journal, Neurotoxicology, ${ }^{11}$ dedicated to the memory of George C. Cotzias, a pioneer in the area of manganese metabolism and its relation to extrapyramidal disorders, presented a series of papers based on presentations at the First Manganese Neurotoxicity Symposium. In his keynote address to this meeting, the late André Barbeau challenged the idea that manganism is an experimental model for Parkinson's Disease and proposed that manganese may be one of many "triggering factors" acting at the dopamine synapse to increase dopamine turnover. With the current renewed interest in manganese neurotoxicity and the urgent need to determine at what levels long term exposure to manganese hinders brain functions, the time is ripe to hold a Second Manganese Neurotoxicity Symposium that would bring together the findings from current research in all areas of manganese neurotoxicity.

\section{REFERENCES}

1. Carl GF, Keen CL, Gallagher BB, et al. Association of low blood manganese concentrations with epilepsy. Neurology 1986; 36 : 1584-1587.

2. Wolters EC, Huang CC, Clark $\mathrm{C}$, et al. Positron emmision tomography in manganese intoxication. Ann Neurol 1989; 26: 647-65lé.

3. Huang CC, Lu CS, Chu NS, et al. Progression after chronic manganese exposure. Neurology 1993; 43: 1479-1483.

4. Lu CS, Huang CC, Chu NS, Calne DB. Levodopa failure in chronic manganism. Neurology 1994; 44: 1600-1602.

5. Iregren A. Using psychological tests for early detection of neurotoxic effects of low level manganese exposure. Neurotoxicology 1994; 15: 671-678.

6. Eriksson H, Gillberg PG, Aquilonius S-M et al. Receptor alterations in manganese intoxicated monkeys. Arch Toxicol 1992; 66: 359-364.

7. Hauser RA, Zesiewicz TA, Martinez C, Rosemurgy AS, Olanow $\mathrm{CW}$. Blood manganese correlates with brain magnetic resonance imaging changes in patients with liver disease. Can J Neurol Sci 1996; 23: 95-98.

8. Krieger D, Krieger S, Jansen O, et al. Manganese and chronic hepatic encephalopathy. Lancet 1995; 346: 270-274.

9. Mergler D, Huel G, Bowler R et al. Nervous system dysfunction among workers with long-term exposure to manganese. Environmental Research 1994; 64: 151-180.

10. Nelson K, Golnick J, Korn T, Angle C. Maganese encephalopathy: utility of early magnetic resonance imaging. Brit $\mathrm{J}$ Industrial Medicine 1993; 50: 510-513

11. Neurotoxicology. Manganese Special Issue Vol. 5, Number 1, 1984

Donna Mergler Centre pour l'étude des interactions biologiques entre la santé et l'environnement (CINBIOSE)

Université du Québec à Montréal 